\title{
Un caso de hipoglucemia como manifestación del Dumping tardío en una gestante
}

\section{A case of hypoglycemia as a manifestation of late Dumping in a pregnant woman}

\author{
Marcela Herazo, Dereck De la Rosa, Karen Beracaza, José Orozco, \\ Lecys Pacheco • Barranquilla (Colombia)
}

\begin{abstract}
Resumen
Primigestante de 26 años edad con antecedente de cirugía bariátrica en Y de Roux, que ingresa al servicio de urgencias por episodios de dolor abdominal y emesis posterior a la ingesta, seguidos de síncope en el contexto de hipoglucemia $<50 \mathrm{mg} / \mathrm{dL}$. Se descartan otras causas de hipoglucemia relacionadas a través del perfil endocrino y se concluye que se trata de un síndrome de Dumping tardío.

Palabras clave: hipoglucemia, embarazo, cirugía bariátrica, síndrome de Dumping.
\end{abstract}

\begin{abstract}
Case of a 26-year-old primipapous with a history of bariatric surgery in Roux-en-Y who was admitted to the emergency department for episodes of abdominal pain and emesis after ingestion followed by syncope, in the context of hypoglycaemia $<50 \mathrm{mg} / \mathrm{dl}$. Other causes of hypoglycemia related through the endocrine profile are ruled out and it is concluded that it is a late Dumping syndrome.

Key words: hypoglycemia, pregnancy, bariatric surgery, Dumping syndrome.
\end{abstract}

Dra. Marcela Herazo Peñaranda: Residente Medicina Interna de la Universidad del Norte, Barranquilla (Atlántico); Dr. Dereck De La Rosa: Especialista en Medicina Interna, Coordinador del Programa Medicina Interna de la Universidad del Norte; Dra. Karen Beracaza: Residente Medicina Interna de la Universidad del Norte; Dr. José Orozco Niño: Médico Interno Universidad del Norte; Dra. Lecys Pacheco Escorcia: Médica Interna Universidad del Norte. Barranquilla (Colombia).

Correspondencia: Dra. Marcela Herazo, Barranquilla (Colombia).

E-mail: marcelaherazo@gmail.com

\section{Introducción}

Se trata de una primigestante con antecedente de cirugía bariátrica, que cursa con episodios repetitivos de síntomas gastrointestinales e hipoglucemia posingesta como consecuencia de un síndrome de Dumping tardío. Lo que resultó en un reto clínico dado que los síntomas aparecen a los cinco años de la intervención y su condición de gestante sumado a hipoglucemia severa limitó los tests de provocación sugeridos para el diagnóstico. Así que ceñidos a la clínica y habiéndose descartado otras causas de hipoglucemia en la paciente, se concluye cursaba con un síndrome de Dumping.

La cirugía bariátrica es cada vez más frecuente en la población joven y la mala adherencia a las recomendaciones médicas provoca complicaciones inherentes al procedimiento.

Son escasos los reportes de casos de Dumping en la gestación y hay una tendencia a subestimar la implicación sobre el bienestar maternofetal, puesto que dan prioridad al riesgo de diabetes gestacional y macrosomía fetal sobre los efectos indeseables como la hipoglucemia al aumentar los requerimientos metabólicos durante el embarazo.

Si bien, la piedra angular son las modificaciones dietarias, existen alternativas de tratamiento, en casos de hipoglucemia de difícil manejo como el caso en mención.

\section{Presentación del caso}

Presentamos el caso de una primigestante de 26 años de edad con 16.6 semanas de gestación por amenorrea, que ingresa a urgencias por cuadro clínico consistente en dolor abdominal tipo cólico que localiza en epigastrio irradiado a mesogastrio de intensidad $5 / 10$, que se inicia posterior a la ingesta de alimentos seguido de náuseas y vómitos de contenido gástrico en tres ocasiones y mareo, por lo que decide consultar; al ingreso síncope asociado a glucemia capilar de $50 \mathrm{mg} / \mathrm{dL}$. En los antecedentes resalta cirugía bariátrica con reconstrucción en Y de Roux hacía cinco años con un asa de $60 \mathrm{~cm}$, asma controlada con última crisis hace cinco años y en farmacológicos: calcio, ácido fólico y sulfato ferroso recomendado durante la gestación; en la revisión por sistemas refería hace una semana episodio de similares características. Durante la exploración física exhaustiva posterior a la administración de bolo de glucosa, la paciente no tenía ningún dato relevante.

Se decide dejar en observación para evaluar su evolución posterior a solución con dextrosa; sin embargo, la paciente presenta varios episodios de hipoglucemia sintomática durante su estancia que obliga a iniciar infusión continua de dextrosa a $3 \mathrm{gr} / \mathrm{h}$. 
Tabla 1. Resultados de perfil endocrinológico.

\begin{tabular}{|c|c|c|c|c|}
\hline HbA1c* & Glucosa & Glucosa post & Cortisol urinario (24h)** & Cortisol 8am \\
\hline $5.3 \%$ & $73 \mathrm{mg} / \mathrm{dL}$ & $71 \mathrm{mg} / \mathrm{dL}$ & $51 \mathrm{mcg} / 24 \mathrm{~h}$ & $33 \mathrm{mcg} / \mathrm{dL}$ \\
\hline Insulina basal & Insulina post & Ac Insulina $* * *$ & Péptido C & ACTH \\
\hline $16 \mathrm{mcUI} / \mathrm{ml}$ & $50.6 \mathrm{mcUI} / \mathrm{ml}$ & $5.6 \mathrm{uUI} / \mathrm{ml}$ & $2.3 \mathrm{ng} / \mathrm{dL}$ & $10 \mathrm{pg} / \mathrm{ml}$ \\
\hline *Hemoglobina glicosilada. **Cortisol urinario*** Anticuerpos antiinsulina. & \\
\hline
\end{tabular}

Se plantea como primera posibilidad diagnóstica el síndrome de Dumping; sin embargo, la gestación e hipoglucemia severa contraindicaban el test de provocación, por lo que se recurre a descartar otras causas potenciales de los síntomas y se solicita el perfil endocrinológico, así como ecografía de abdomen total y pruebas de bienestar fetal (ecografía obstétrica).

Se reciben paraclínicos dentro de la normalidad cuyos valores se encuentran en la Tabla 1. La ecografía de abdomen total no mostró cambios en la morfología pancreática y la obstétrica mostró bienestar fetal.

Teniendo en cuenta los resultados y habiéndose descartado con ello otras causas de hipoglucemia y ante la recurrencia de su clínica en asocio con la ingesta, así como la dificultad para el destete del aporte de dextrosa endovenosa, se considera que la paciente está cursando con síndrome de Dumping tardío.

Se procede a intervenir la dieta de la paciente aumentando la proporción de proteínas, reemplazando los carbohidratos simples por complejos, fraccionando la dieta a cada dos horas y recomendando que las bebidas se ingirieran distante de los sólidos. Con lo que se logra una adecuada respuesta metabólica permitiendo el alta hospitalaria de la paciente; sin embargo, tuvo dos recurrencias que requirieron nuevos ingresos. Finalmente, se logró llevar a término la gestación, sin complicaciones maternofetales relevantes hasta la fecha.

\section{Discusión}

La cirugía bariátrica en mujeres en edad fértil ha dado lugar a la aparición de una población particular de mujeres cuyos embarazos deben ser considerados y tratados como de alto riesgo (1), puesto que si bien hay claro impacto en la disminución de los trastornos hipertensivos durante el embarazo, la diabetes gestacional y la macrosomía fetal, también aumenta el porcentaje de fetos pequeños para la edad gestacional y prematuridad (2).
El caso de nuestra gestante, nunca fue previsto como de alto riesgo durante los primeros controles prenatales y tampoco fueron estimados los requerimientos metabólicos que son mayores conforme aumenta la edad gestacional. Razón suficiente para desencadenar los síntomas del Dumping tardío, los cuales habían permanecido silentes durante los cinco años previos.

El tiempo de presentación de los síntomas tan distantes a la cirugía bariátrica, constituyó por ende un factor de confusión. Las limitaciones del diagnóstico temprano estuvieron ligadas a la presentación tardía de los síntomas y a la contraindicación absoluta de la realización del test de provocación; en cuanto al manejo hospitalario, fue dispendioso lograr un buen control metabólico, con dos recurrencias a su egreso ya habiendo intervenido la dieta de la paciente.

En la actualidad hay opciones terapéuticas para el tratamiento de estos pacientes que responden pobremente a las medidas no farmacológicas, tales como el uso de la acarbosa y los análogos de la somatostatina, así como las quirúrgicas con las que se busca reducir el tamaño del asa o restablecer la anatomía, estas últimas en casos refractarios (3).

Hay pobre evidencia sobre el tratamiento del Dumping refractario en las gestantes. La acarbosa y los análogos de la somatostatina han sido clasificados como categoría B por la FDA; sin embargo, no se encuentra en la literatura estudios que avalen su uso en este grupo de pacientes.

\section{Referencias}

1. Barrera C H. Embarazo después de cirugía bariátrica. Rev Méd Clín Condes. 2014; 25 (6): 944-51.

2. Johansson K, Cnattingius, S, Näslund, I, Roos, N, Lagerros YT, Granath F, et al. Outcomes of Pregnancy After Bariatric Surgery. (2015). New N Engl J Med. 2015; (372): 814-24.

3. van Beek AP, Emous M, Laville M, Tack J. Dumping syndrome after esophageal, gastric or bariatric surgery: pathophysiology, diagnosis, and management. Obes Rev. 2017; 18 (1): 68-85. doi: 10.1111/obr.12467. 